\title{
Abundance of Diseases in Food Fishes of North East Region of India With Reference To Economic Loss
}

\author{
Haren Ram Chiary ${ }^{1}$, Kapinder ${ }^{1}$, Umesh C. Goswami ${ }^{2}$, H.S. Singh ${ }^{3}$ \\ ${ }^{I}$ (Department of Zoology, Kirori Mal College, University of Delhi, Delhi-110007, India) \\ ${ }^{2}$ (Department of Zoology, Gauhati University, Guwahati-781014, Assam) \\ ${ }^{3}$ (Department of Zoology, Ch. Charan Singh University, Meerut - 250004, India)
}

\begin{abstract}
Aquaculture is the fastest growing food producing sector in the world. The inland fishery resources of India have a rich production potential. North Eastern Region of the country is located between $21.570 \mathrm{~N}-$ 29.300N latitude and 89.460E - 97.300E longitude. The fishery resources of the region fall within all three types of climate i.e. tropical, sub-tropical and temperate and represent a strong biodiversity. Two principal rivers Brahmaputra, Barak and their numerous tributaries harbor varieties of fish species. The threat of diseases has now become a risk to the growth of the aquaculture sector which significantly affects both economic and socio economic development. A list of 197 species and 25 families of fish is given in the paper with the diseases reported in them.
\end{abstract}

Key words: Diseases, Inland fishery, North East region, Threat.

\section{Introduction}

The North East region has about 19,150 km of rivers; 23,972 ha. of reservoirs; 1,43,740 ha.of lakes; 40,809 ha. of ponds and 2,780 ha. of rice-cum-fish culture area. The State Assam which forms about 30\% of the North Eastern region has Brahmaputra and Barak river systems and their numerous tributaries (combined length 4820 $\mathrm{km})$ and a large number of flood plain wet lands (Beel).The North East India is considered as one of the hot spots of fresh water fish biodiversity in the world (Kottelat and Whitten, 1996).

The North Eastern Region shares its fish fauna predominantly with that of the Indo-Gangetic fauna and to a small extent with the Burmese and South China fish fauna (Yadav and Chandra, 1994). Goswamiet al.(2012) listed 422 fish species from north east India, belonging to 133 genera and 38 families. The seven North Eastern states of India: Arunachal Pradesh, Assam, Manipur, Meghalaya, Mizoram, Nagaland and Tripura are blessed with diversified fish fauna. The region has been ranked sixth among the top 25 biodiversity spots in the world. The region has about 19,150 km of rivers; 23,972 ha. of reservoirs; 1,43,740 ha.of lakes;40,809 ha. of ponds and 2,780 ha.of rice-cum-fish culture area (Kiran: A report by ICAR). A list of Rivers, Lakes, and waterfalls of the north east region is given below:

\section{Rivers}

Bhogdoi, Siang/ Dihang/ Brahmaputra, Dhansiri, Dihing, Diphlu, Feni, Gumti, Haor, Iril, Kaladan, Kameng, Bhoreli, Karnaphuli, Khowai, Kolong, Lohit, Manas, Manu, Mora Dhansiri, Muhuri, Myntdu, Mora diphlu, Sankosh or Puna Tsang Chhu, Simsang or Someshwari, Surma, Thega River, Tirap.

Lakes

Kamalasagar, Lohtak and Umiam.

Waterfalls

Bishop, Kynrem, Langshiang.

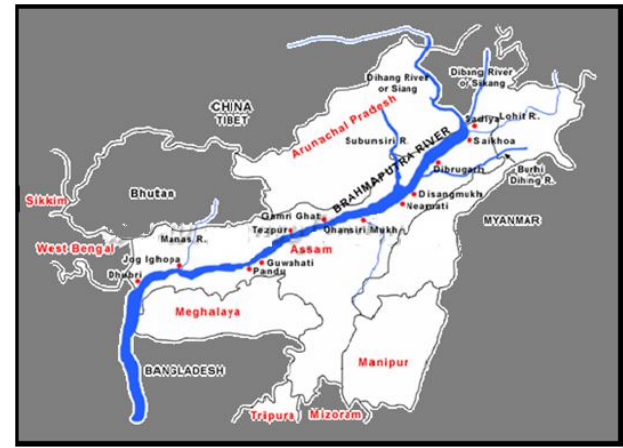

Fig.1-Map showing rivers and tributaries flowing in North East region.

Riverine fishery resources of the states of North East region have diversified fish fauna but still the old traditional methods of fishing are being used and primitive and outdated practices are followed for fishing. A large number of diseases occur in fishes, due to nutritional deficiency or unhygienic condition of water or attack 
of parasites. Among the parasites that infect freshwater fishes, helminths- represented by 5 taxonomic units i.e. Monoginoidea, Trematoda, Cestoda, Nematoda and Acanthocephala,they often cause veterinary problems worldwide (Williams and Jones, 1994)

List of some common parasites of fishes is given below (S.S. Khanna and H.R. Singh)

Costia

Flagellated Protozoan

Ichthyophthirius

Ciliated Protozoan

Trichodina

Ciliated Protozoan

Lernea

Copepod Crustacean

Argulus

Ergasilus

Crustacean

Cercaria of Bucephalus

Diplozoon

Copepod

Trematode

Monogenea

Gyrodactylus

Dactylogyrus

Saprolegnia

Monogenea

Monogenea

Fungus

It must to be mentioned that reports of fish mortalities occurring in the water bodies of India are infrequent. Fisheries management practices are not working properly in the region. However, with the outbreak of epizootic ulcerative syndrome (a disease caused by the fungus,Aphanomycesinvadans, red spots develop on the skin which later on becomes ulcers and then followed by the development of granulomas on the internal organs which becomes the cause of mortality in fishes) a system of disease surveillance and monitoring was initiated in a structured manner, and reports have been obtained.

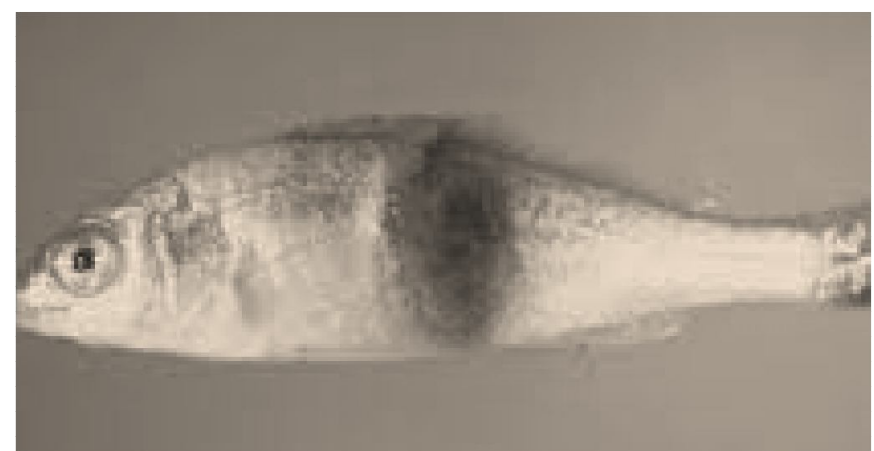

Fig. 2-EUS (Epizootic Ulcerative Syndrome) disease in fish

Table 1:Data for outbreaks of EUS in India.

\begin{tabular}{|c|c|c|c|}
\hline \multicolumn{2}{|c|}{$\begin{array}{l}\text { Range in Prevalence (\%) } \\
\text { by Affeeted Fish Species }\end{array}$} & \multicolumn{2}{|c|}{$\begin{array}{c}\text { Range in Prevalence (\%) by } \\
\text { Geographic Area } \\
\text { (in All Species Affected) }\end{array}$} \\
\hline Species & Prevalence & State & Prevalence \\
\hline Channa spp. & $20-100$ & Assam & $30-60$ \\
\hline Puntius spp. & $5-100$ & Tripura & $35-70$ \\
\hline Glossogobius spp. & $10-60$ & Meghalaya & $10-35$ \\
\hline Mystus sp. & $5-75$ & West Bengal & $15-65$ \\
\hline Notopterus spp. & $3-25$ & Bihar & $20-30$ \\
\hline Wallago attu & $7-20$ & Orissa & $20-45$ \\
\hline Mastacembelus spp. & $10-35$ & Uttar Pradesh & $15-20$ \\
\hline Anabas testudineus & $10-55$ & Tamil Nadu & $5-25$ \\
\hline Amblypharyngodon mola & $5-10$ & Maharashtra & $5-10$ \\
\hline Rhinomugit corsula & $1-5$ & Kerala & $30-65$ \\
\hline Clarias batrachus & $10-30$ & & \\
\hline Heteropneustes fossilis & $10-20$ & & \\
\hline Catia catia & $5-15$ & & \\
\hline Labeo rohita & $5-10$ & & \\
\hline Cirhinus cirhosus & $5-20$ & & \\
\hline Cyprinus carpio & $10-25$ & & \\
\hline Ctenopharyngodon idellus & $2-5$ & & \\
\hline
\end{tabular}


Table 2: A list of food species of North east region and the diseases reported is given below

\begin{tabular}{|c|c|c|}
\hline Family & Name of Fishes & Diseases \\
\hline \multirow[t]{2}{*}{ Anabantidae } & 1. Anabas cobojiu & DD \\
\hline & 2. Anabas testudineus & $\begin{array}{l}\text { Anchor worm Disease, Procerovum Infestation, Gnathostoma Infestation, Procerovum Infestation, Centrocestus } \\
\text { Infestation, Camallanus Infection, Stellantchasmus Infestation, Lernaea Infestation, Dactylogyrus Gill Flukes Disease, } \\
\text { Contracaecum Disease, Tripartiella Disease, Gnathostoma Infestation, Camallanus Infection, Allocreadium Infestation, } \\
\text { Neopecoelina Infestation, Camallanus Infection, Paragendria Infestation, Lernaea Infestation, Parasitic infestations } \\
\text { (protozoa, worms, etc.) }\end{array}$ \\
\hline \multirow[t]{3}{*}{ Anguillidae } & 1. Anguilla bengalensis & $\mathrm{DD}$ \\
\hline & 2. Gonialosamanmina & DD \\
\hline & 3. Gudusiachapra & DD \\
\hline \multirow[t]{24}{*}{ Bagridae } & 1. Batasiofasciolatus & DD \\
\hline & 2. Batasioniger & DD \\
\hline & 3. Batasiospilurus & DD \\
\hline & 4. Batasiotengana & DD \\
\hline & 5. Chandramarachandramara & DD \\
\hline & 6. Hemibagrusmenoda & DD \\
\hline & 7. Hemibagrusmicrophthalmus & Gnathostoma Infestation, Parasitic infestations (protozoa, worms, etc.) \\
\hline & 8. Hemibagruspeguensis & DD \\
\hline & 9. Mystusarmatus & DD \\
\hline & 10. Mystusbleekeri & DD \\
\hline & 11. Mystuscavasius & $\begin{array}{l}\text { Procamallanus Infection, Isoparorchis Infestation, Allocreadium Infestation, Buckleynema Disease,Cucullanus Disease, } \\
\text { Parasitic infestations (protozoa, worms, etc.) }\end{array}$ \\
\hline & 12. Mystusfalcarius & DD \\
\hline & 13. Mystushorai & $\mathrm{DD}$ \\
\hline & 14. Mystusmontanus & DD \\
\hline & 15. Mystuspulcher & $\mathrm{DD}$ \\
\hline & 16. Mystusrufescens & $\mathrm{DD}$ \\
\hline & 17. Mystustengara & $\begin{array}{l}\text { Euclinostomum Infestation, Procamallanus Infection, Procamallanus Disease, Isoparorchis Infestation, } \\
\text { Neoechinorhynchus Disease, Allocreadium Infestation, Procamallanus Infection, Parasitic infestations (protozoa, } \\
\text { worms, etc.) }\end{array}$ \\
\hline & 18. Mystusvittatus & $\begin{array}{l}\text { Procamallanus Infection, Isoparorchis Infestation, Pallisentis Disease, Palaeorchis Disease, Buckleynema Disease, } \\
\text { Neoechinorhynchus Infestation, Parasitic infestations (protozoa, worms, etc.), Aphanomyces Infestation, Saprolegnia } \\
\text { Infestation, Saprolegnia Infestation (Fungal diseases) }\end{array}$ \\
\hline & 19. Olyraburmanica & DD \\
\hline & 20. Rama rama & DD \\
\hline & 21. Rita rita & $\begin{array}{l}\text { Contracaecum Disease, Cucullanus Disease, Opisthorchis Disease, Phyllodistomum Infestation, Rhabdochona } \\
\text { Infestation, Parasitic infestations (protozoa, worms, etc.) }\end{array}$ \\
\hline & 22. Sperataacicularis & $\mathrm{DD}$ \\
\hline & 23. Sperataaor & $\begin{array}{l}\text { Isoparorchis Infestation, Bucephalus Infestation, Bucephalus Disease, Prosorhynchoides Disease, Coitocaecum Disease, } \\
\text { Caryophyllaeus Infestation, Contracaecum Infestation, Paragendria Disease, Pingus Infestation, Parasitic infestations } \\
\text { (protozoa, worms, etc.) }\end{array}$ \\
\hline & 24. Sperataseenghala & DD \\
\hline Chacidae & 1. Rhinomugilcorsula & DD \\
\hline \multirow{2}{*}{ Channidae } & 1. Channaamphibeus & $\mathrm{DD}$ \\
\hline & 2. Channaaurantimaculata & $\mathrm{DD}$ \\
\hline
\end{tabular}


3. Channabarca

Channableheri

Channagachua

Channahatcourtbutler

Channamarulius

8. Channaorientalis

Channapunctata

Channastewartii

11. Channastriata
DD

Genarchopsis Infestation, Pallisentis Disease, Camallanus Infection, Parasitic infestations (protozoa, worms, etc.)

Camallanus Disease, Contracaecum Disease, Phyllodistomum Infestation, Neocamallanus Infestation, Isoparorchis Infestation, Pallisentis Disease, Pallisentis Infestation, Allogomtiorema Infestation, Paracamallanus Infection, Crowcrocaecum Infestation, Anchistrocephalus Disease, Bothriocephalus Infestation, Polyonchobothrium Disease, Taphrobothrium Infestation, Heliconema Infestation, Neocamallanus Disease, Pallisentis Infestation, Parasitic infestations (protozoa, worms, etc.)

DD

Anchor worm Disease, Trichodinosis, Contracaecum Disease, Neocamallanus Infestation, Procamallanus Disease, Euclinostomum Infestation, Ascaris Disease, Isoparorchis Infestation, Genarchopsis Infestation, Pallisentis Disease, Palaeorchis Disease, Allocreadium Infestation, Phyllodistomum Infestation, Ascaridia Disease, Pallisentis Infestation, Euclinostomum Infestation, Neopecoelina Infestation, Neopecoelina Disease, Philopinna Disease, Pallisentis Infestation, Anchistrocephalus Disease, Neocamallanus Disease, Pallisentis Infestation, Camallanus Infection, Eucreadium Infestation, Parasitic infestations (protozoa, worms, etc.)

Anchor worm Disease, Fish louse Infestation, Trichodinosis, Skin Flukes, Yellow Grub, Camallanus Disease, Cercaria Disease, Clinostomum Infestation (metacercaria), False Fungal Infection (Apiosoma sp.), False Fungal Infection (Epistylis sp.), Haplorchis Infestation, Procerovum Infestation, Posthodiplostomum Infestation, Turbidity of the Skin (Freshwater fish), Anchorworm Disease (Lernaea sp.), Haplorchis Infestation, Clinostomoides Infestation, Neodiplostomum Disease, Opegaster Infestation, Gnathostoma Infestation, Neocamallanus Infestation, Centrocestus Infestation, Fish Louse Infestation, Clinostomum Infestation, Euclinostomum Infestation, Posthodiplostomum Disease, Piscicola Infestation (Piscicola sp.), Fish louse Infestation, Contracaecum Disease, Phyllodistomum Disease, Acanthogyrus Infestation, Gnathostoma Infestation, Neocamallanus Infestation, Euclinostomum Infestation, Procamallanus Infection, Isoparorchis Infestation, Pallisentis Disease, Procamallanus Infection, Echinocephalus Procamallanus Infection, Isoparorchis Infestation, Pallisentis Disease, Procamallanus Infection, Echinocephalus Disease, Pallisentis Infestation, Euclinostomum Infestation, Pallisentis Infestation, Allogomtiorema Infestation,
Paracamallanus Infection, Anchistrocephalus Disease, Bothriocephalus Infestation, Polyonchobothrium Disease, Paracamallanus Infection, Anchistrocephalus Disease, Bothriocephalus Infestation, Polyonchobothrium Disease, Taphrobothrium Infestation, Neocamallanus Disease, Pallisentis Infestation, Lernaea Infestation, Camallanus Infection,
Parasitic infestations (protozoa, worms, etc.), Epizootic Ulcerative Syndrome, Epizootic Ulcerative Syndrome, (Viral Parasitic infestations (protozoa, worms, etc.),
diseases), Aeromonosis, Bacterial diseases.

Yellow Grub, Dactylogyrus Gill Flukes Disease, Trichodinosis, Dactylogyrus Gill Flukes Disease, Trichodinosis, Skin Flukes, Cichlidogyrus Disease, Cryptobia Infestation, False Fungal Infection (Apiosoma sp.), False Fungal Infection (Epistylis sp.), Transversotrema Infestation, Caligus Infestation, Alitropus Infestation, Trichodina Infection, Tripartiella Infestation, Trichodina Infection, Cristaria Infestation, Cichlidogyrus Infestation, Ichthyophthirius Disease, Tripartiella Infestation, Cichlidogyrus Infestation, Cichlidogyrus Infestation, Cichlidogyrus Infestation, Trichodina Infestation, Trichodina Infestation, Trichodina Infestation, Enterogyrus Infestation, Gyrodactylus Infestation, Gyrodactylus Infestation, Lamproglena Infestation, Fish louse Infestation, Turbidity of the Skin (Freshwater fish), Ergasilus Disease, Trypanosoma Infection, Sporozoa-infection (Myxobolus sp.), Contracaecum Disease (larvae), Fish Tuberculosis, Amplicaecum Infection (Larvae), Cichlidogyrus Infestation, Gnathostoma Disease (larvae), Tuberculosis, Amplicaecum Infection (Larvae), Cichlidogyrus Infestation, Gnathostoma Disease (larvae),
Cichlidogyrus Infestation, Cichlidogyrus Disease, Acanthogyrus Infestation, Cichlidogyrus Infestation, Cichlidogyrus Cichlidogyrus Infestation, Cichlidogyrus Disease, Acanthogyrus Infestation, Cichlidogyrus Infestation, Cichlidogyrus
Infestation, Cichlidogyrus Infestation, DilepidCestode larvae Infestation (general sp.), Cichlidogyrus Infestation, Trypanosoma Infestation, Dactylosoma Infection, Parasitic infestations (protozoa, worms, etc.), Aeromonosis, Myxobacterial Infections, Aeromonosis, Edwardsiellosis, Epitheliocystis (Bacterial diseases), Iridovirosis, Whirling Viral Disease of Tilapia Larvae, (Viral diseases).

Trichodinosis, Piscicola Infestation (Piscicola sp.), Sanguinicola Disease, Fish Louse Infestation, Procamallanus Infection, Dolops Infestation, Chilodonella Infection, Henneguya Infection, Gyrodactylus Infestation, Phyllodistomum Infestation, Neodactylogyrus Infestation, Astiotrema Infestation, Quadriacanthus Infestation, Polyonchobothrium 
3. Heteropneustesfossilis

Clupeidae

1. Gudusia variegate

Nematalosanasus

3. Tenualosailisha

Cobitidae

Cyprinidae

$\begin{array}{ll}\text { 1. } & \text { Acantopsismultistigmatus } \\ \text { 2. } & \text { Pangiopangia } \\ \text { 3. } & \text { Pangiolongipinnis } \\ \text { 1. } & \text { Amblypharyngodonmola } \\ \text { 2. } & \text { Aspidopariajaya } \\ \text { 3. } & \text { Aspidopariamorar } \\ \text { 4. } & \text { Aspidopariaukhrulensis } \\ \text { 5. } & \text { Banganadero } \\ \text { 6. } & \text { Banganadevdevi } \\ \text { 7. } & \text { Bariliusbakeri } \\ \text { 8. } & \text { Bariliusbarila } \\ \text { 9. } & \text { Bariliusbarna } \\ \text { 10. } & \text { Bariliusbarnoides } \\ \text { 11. } & \text { Bariliusbendelisis } \\ \text { 12. } & \text { Bariliuschatricensis } \\ \text { 13. } & \text { Bariliusdimorphicus } \\ \text { 14. } & \text { Bariliusdogarsinghi } \\ \text { 15. } & \text { Bariliusgatensis } \\ \text { 16. } & \text { Bariliuslairokensis } \\ \text { 17. } & \text { Bariliusngawa } \\ \text { 18. } & \text { Bariliusradiolatus } \\ \text { 19. } & \text { Bariliusshacra } \\ \text { 20. } & \text { Bariliustileo } \\ \text { 21. } & \text { Bariliusvagra } \\ \text { 22. } & \text { Chaguniuschagunio } \\ \text { 23. } & \text { Chaguniusnicholsi } \\ \text { 24. } & \text { Chela cachius } \\ \text { 25. } & \text { Chela fasciatus } \\ \text { 26. } & \text { Chela khujairokensis } \\ \text { 27. } & \text { Chela laubuca } \\ \text { 28. } & \text { Cirrhiniusariza } \\ \text { 29. } & \text { Cirrhinius cirrhosis } \\ \text { 30. } & \text { Cirrhinusmrigala } \\ \text { 31. } & \text { Cirrhinusreba } \\ \text { 32. } & \text { Crossocheilusburmanicus } \\ \text { 33. } & \text { Crossocheiluslatius } \\ \text { 34. } & \text { Ctenopharyngodonidella }\end{array}$

Infestation, Macrogyrodactylus Infestation, Parasitic infestations (protozoa, worms, etc.)

Fish louse Infestation, Intestinal Ligulosis, Dactylogyrus Gill Flukes Disease, Yellow Grub, Contracaecum Disease, Clinostomum Infestation (metacercaria), Orientocreadium Infestation, Gnathostoma Infestation, Procamallanus Infection Procamallanus Disease, Euclinostomum Infestation, Procamallanus Infection Procamallanus Disease, Genarchopsis Infestation, Macrolecithus Disease, Palaeorchis Disease, Allocreadium Infestation, Procamallanus Infection, Lytocestus Disease, Pseudocaryophyllaeus Infestation, Pseudocaryophyllaeus Disease, Ascaridia Disease, Allocreadium Infestation, Aphallus Disease, Euclinostomum Disease, Eumasenia Disease, Macvicaria Infestation, Neopecoelina Infestation, Neopecoelina Disease, Opegaster Infestation, Philopinna Disease, Bialovarium Disease, Lernaeocera Disease (Lernaeocera sp.), Parasitic infestations (protozoa, worms, etc.).

$$
\begin{aligned}
& \text { DD } \\
& \text { DD }
\end{aligned}
$$

Aphanurus Infestation, Faustula Infestation, Lecithocladium Infestation, Lecithocladium Infestation, Gymnorhynchus Disease, Ilisha Infestation, Poecilancistrum Infestation, Pterobothrium Infestation, Pallisentis Disease, Parasitic infestations (protozoa, worms, etc.).

DD

DD

DD

DD

DD

DD

DD

DD

DD

DD

DD

DD

DD

DD

DD

DD

$\mathrm{DD}$

DD

DD

DD

DD

DD

DD

DD

DD

DD

DD

$\mathrm{DD}$

DD

DD

DD

DD

DD

Fish louse Infestation, Myxidium Infection, Trichodina Infection, Tripartiella Infestation, Bothriocephalus Infestation, 
35. Cyprinionsemiplotum

36. Cyprinuscarpio

37. Daniojaintianensis

38. Garraflavatra

39. Garragotyla

40. Garralissorhynchus

41. GarraRupecula

42. Gibelioncatla

43. Gymnocyprisdobula

44. Gymnocyprisscleracanthus

45. Horalabiosajoshuai

46. Hypophthalmichthysmolitrix

47. Hypophthalmichthysnobilis
48. Labeoangra
49. Labeoariza
50. Labeobata
51. Labeoboga
52. Labeoboga
53. Labeocalbasu
54. Labeodyocheilus
54. Labeofimbriatus
55. Labeogonius
57. Labeonandina
57. Labeopangusia
58. Labeorohita

59. Megarasboraelanga

60. Neolissochilusblythii

61. Neolissochilushexagonolepis
Myxobolus Infection, Fish louse Infestation, Dactylogyrus Gill Flukes Disease, Trichodinosis, Sporozoa-infection (Myxobolus sp.), Anchorworm Disease (Lernaea sp.), Piscinoodinium Infection, Capillaria Infestation, Gonad Nematodosis Disease, Spiroxys Infestation, Sanguinicola Infection, Anchor worm Disease, Parasitic infestations (protozoa, worms, etc.), Water mold Disease, Fungal diseases, Columnaris Disease, Bacterial diseases, SVC, Grass Carp Picornavirus, Grass Carp Haemorrhagic, Unclassifed Grass Carp Virus (Viral diseases).

DD

Anchor worm Disease, Fish louse Infestation, Coccidiosis (intestine), Boil Disease, Costia Disease, Dactylogyrus Gill Flukes Disease, Fish leech Infestation, Trichodinosis, Skin Flukes, Chilodonella Disease, Worm Cataract, White spot Disease, Trichodinosis, Skin Flukes, Trichodinella Infection, Trichodina Infection, Myxobolus Infection, Thelohanellus Infection, Anchorworm Disease (Lernaen sp), Dactylogyrus Gill Flukes Disease, Trichodinosis, Turbidity of the Skin (Fresich infestaton, Capillation Infection, Velvet Disease (protozoa, worms, etc.), Fin-ot Disease (late stage), Red spot Disease, Fin Rot (early stage), Columnaris Disease, Aeromonosis, Infectious ascites (Ornament.), Bacterial Infections (general), Enteric Redmouth Disease, Edwardsiellosis, Epitheliocystis, (Bacterial diseases), Fungal Gill Rot (sanguinis), Water mold Disease (Fungal
diseases), Lymphocystis Disease, Lymphocystis Disease (dark), Koi Herpes Virus, Fish Pox Disease, Fish Pox Disease, diseases), Lymphocystis Disease, Lymphocystis Disease (dark), Koi Herpes Virus, Fish Pox Disease, Fish Pox Disease,
Carp Iridovirus, Carp Coronovirus Infection, Carp Reovirus, (Viral diseases), Unspecified tumors, Neoplasia (tumors of unknown origin), Congenital Deformities, Others.

DD

$\mathrm{DD}$

DD

DD

DD

DD

DD

Bothriocephalus Infestation, Myxobolus Infection, Myxobolus Infection, Anchorworm Disease (Lernaea sp.), Dactylogyrus Gill Flukes Disease, Trichodinosis, Anchorworm Disease (Lernaea sp.), Parasitic infestations (protozoa, worms, etc.), Enteric Redmouth Disease (Bacterial diseases).

White spot Disease, Dactylogyrus Gill Flukes Disease, Trichodinosis, Cryptobia Infestation, False Fungal Infection (Apiosoma sp.), Turbidity of the Skin (Freshwater fish), Myxidium Infection, Trichodina Infection, Tripartiella Infestation, Trichodinella Infection, Ichthyobodo Infection, Bothriocephalus Infestation, Fish Louse Infestation, Piscinoodinium Infection, Sanguinicola Infection, Dactylogyrus Infestation, Anchor worm Disease, Parasitic infestations (protozoa, worms, etc.), Enteric Redmouth Disease (Bacterial diseases), SVC (Viral diseases). DD

Hemiclepsis infestations, Parasitic infestations (protozoa, worms, etc.),

$\mathrm{DD}$

DD

DD

DD

$\mathrm{DD}$

DD

White spot Disease, Fish louse Infestation, Dactylogyrus Gill Flukes Disease, Trichodinosis, Sporozoa-infection (Myxobolus sp.), Ichthyophthirius Disease, Neascus Disease, Thelohanellus Infection, Sachalinorhynchus Disease, Parasitic infestations (protozoa, worms, etc.), Aeromonosis (Bacterial diseases), Water mold Disease (Fungal diseases).

DD 


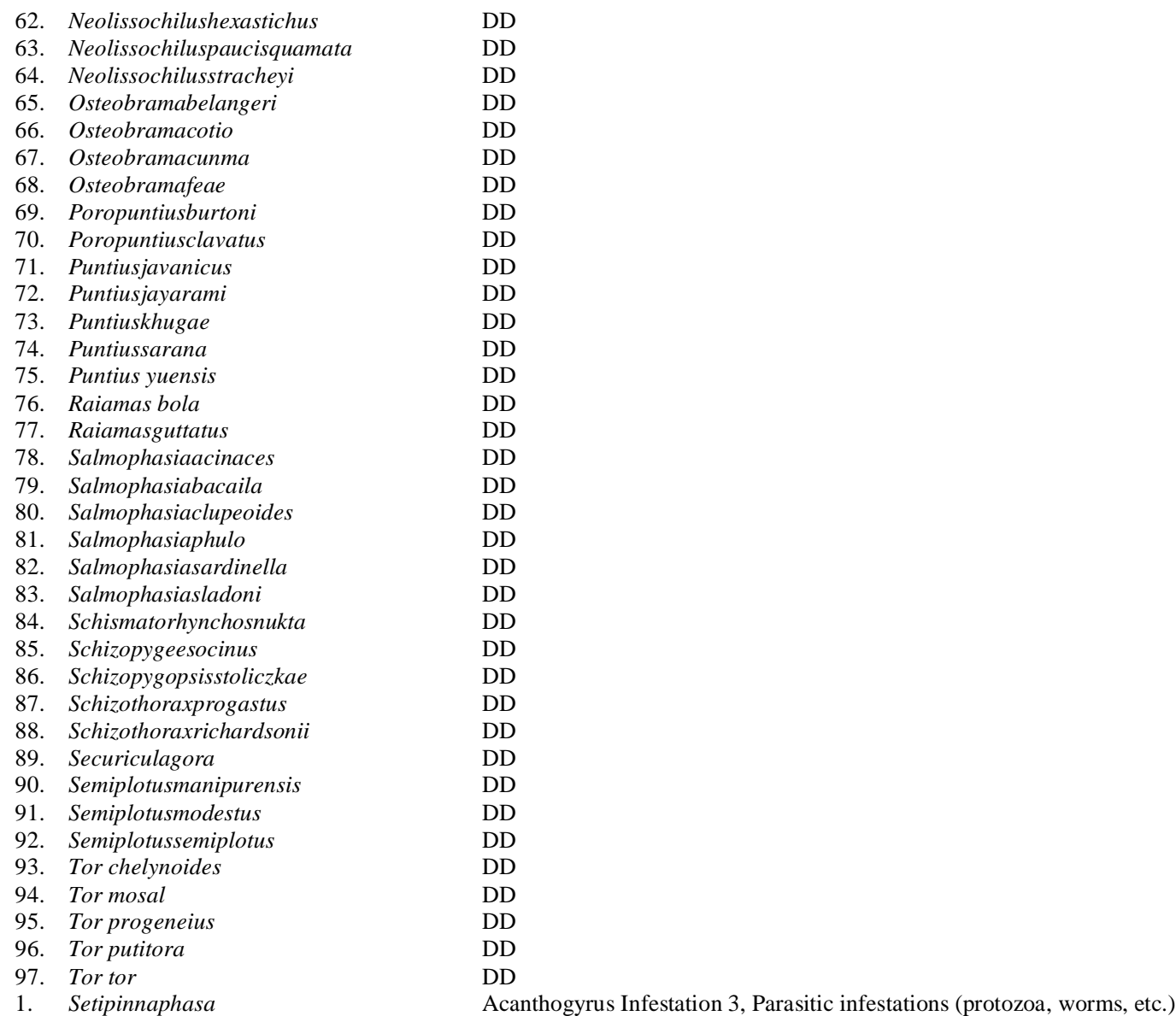

Engraulidae

. Setipinnaphasa

Gobida

2. Glossogobiusgiuris
Acant

Anchor worm Disease, Procerovum Infestation, Posthodiplostomum Infestation, Haplorchis Infestation, Opegaster Infestation, Gnathostoma Infestation, Ergasilus Disease, CentrocestusInfestation, Azygia Infestation, Echinochasmus Infestation, Erilepturus Infestation, Prosorhynchus Infestation, Orientocreadium Disease, Neochasmus Infestation, Carneophallus Infestation, Bothriocephalus Disease, Proteocephalus Disease, Lernaea Infestation, Camallanus Disease, Contracaecum Disease, Capillaria Infestation, Genarchopsis Infestation, Genarchopsis Disease, Pallisentis Disease, Allocreadium Infestation, Phyllodistomum Infestation, Dactylogyrus Infestation, Pallisentis Infestation, Allocreadium Infestation, Allocreadium Infestation, Opegaster Infestation, Opegaster Disease, Pallisentis Infestation, Echinorhynchus Infestation, Tetrarhynchus Disease, Lernaea Infestation, Parasitic infestations (protozoa, worms, etc.).

Anchor worm Disease, Opegaster Infestation, Neochasmus Infestation, Parasitic infestations (protozoa, worms, etc.) 
Rhinomugilcorsula Chitalachitala

Notopterusnotopterus

Ophichthidae

Pangasiid

1. Pisodonophisboro

1. Pangasiuspangasius

Pristigasteridae Psilorhynchidae

Salmonidae

Schilbeidae

Ompokbimaculatus

2. Ompokpabda

3. Ompokpabo

Pterocryptisbarakensis

Pterocryptisberdmore

Pterocryptisindicus

Pterocryptis afghan

Pterocryptistorrentis

Pterocryptisgangelica

(general), Bacterial disease

Contracaecum Disease, Capillaria Infestation, Procamallanus Infection, Isoparorchis Infestation, Allocreadium Infestation, Allocreadium Infestation, Cotylogonoporum Infestation, Paracamallanus Infection, Marsipometra Infestation, Proleptus Infestation, Pseudoproleptus Infestation, Parasitic infestations (protozoa, worms, etc.), Saprolegnia Infestation, Achlya Infestation, Saprolegnia Infestation (Fungal diseases).

$\mathrm{DD}$

DD

Fish louse Infestation, Procamallanus Infection, Pleurogenoides Infestation, Parasitic infestations (protozoa, worms, etc.)

Contracaecum Disease, Gymnorhynchus Disease, Allocreadium Disease, Cucullanus Infestation, Parasitic infestations

(protozoa, worms, etc.)

(pro

DD

DD

DD

DD

DD

DD

Whirling Disease, Hysterothylacium Infection (Hysterothylacium sp.), Proliferative Kidney Disease, Aeromonosis, Bacterial diseases, Camallanus Infection, Camallanus Infection, Parasitic infestations (protozoa, worms, etc.), Enteric Redmouth Disease, Epitheliocystis, (Bacterial diseases).

DD

Contracaecum Disease, Isoparorchis Infestation, Genarchopsis Disease, Palaeorchis Disease, Allocreadium Infestation,

Contracaecum Disease, Procamallanus Disease, Isoparorchis Infestation, Palaeorchis Disease, Caryophyllaeus Infestation, Phyllodistomum Infestation, Spinitectus Infestation, Neocamallanus Infestation, Fish Louse Infestation, Parasitic infestations (protozoa, worms, etc.)

Acanthogyrus Infestation, Procamallanus Infection, Procamallanus Disease, Prosorhynchoides Disease, Prosorhynchoides Infestation, Parasitic infestations (protozoa, worms, etc.)

Ichthyoxenus Infestation, Parasitic infestations (protozoa, worms, etc.).

Contracaecum Disease, Genarchopsis Disease, Gymnorhynchus Disease, Pallisentis Disease, Bucephalus Disease, Caryophyllaeus Infestation, Devendrosentis Infestation, Parasitic infestations (protozoa, worms, etc.).

DD

Contracaecum Disease, Capillaria Infestation, Gnathostoma Infestation, Procamallanus Infection, Isoparorchis Infestation, Gonocera Infestation, Phyllodistomum Infestation, Pallisentis Infestation, Allocreadium Infestation, Pleurogenes Infestation, Eustrongylides Infestation, Falcaustra Infestation, Parasitic infestations (protozoa, worms, etc.).

Fish louse Infestation, Contracaecum Disease, Gnathostoma Infestation, Procamallanus Infection, Euclinostomum Infestation, Procamallanus Infection, Isoparorchis Infestation, Euclinostomum Disease, Caryophyllaeus Infestation, Pleurogenes Infestation, Eustrongylides Disease, Pallisentis Infestation, Parasitic infestations (protozoa, worms, etc.). $\mathrm{DD}$

DD

$\mathrm{DD}$

DD

$\mathrm{DD}$

$\mathrm{DD}$

$\mathrm{DD}$ 
10. Wallagoattu

Sisoridae

Synbranchidae
Contracaecum Disease, Gnathostoma Infestation, Isoparorchis Infestation, Genarchopsis Infestation, Procamallanus Infection, Spinitectus Infestation, Goezia Disease, Parasitic infestations (protozoa, worms, etc.), Epizootic Ulcerative Syndrome (Viral diseases)

\section{Bagariusbagarius} Bagariusyarrelli

Glyptothoraxchimtuipuiensis

Pseudecheneisukhrulensis

Pseudecheneissirenica

Pseudecheneiskoladynae

1. Monopterusalbus

Monopteruscuchia

Monopterushodgarti

Ophisternonbengalense
Rhabdochona Infestation, Opisthorchis Infestation, Parasitic infestations (protozoa, worms, etc.).

$\mathrm{DD}$

DD

DD

DD

Trypanosoma Infection, Pallisentis Infestation, Dentiphilometra Infestation, Proleptinae Disease,Eustrongylides Disease (larvae), Parasitic infestations (protozoa, worms, etc.).

$\mathrm{DD}$

DD

DD 
Impacts of diseases have been estimated in socioeconomic terms like losses in production, income, employement, market access or market share, investment and consumer confidence etc. (Melba, 2005).Inadequate implemented biosecurity measures have led to significant losses due to aquatic animal diseases in many countries around the world. Arthur and Subasinghe, (2002) reported the impact of aquatic diseases on wild population and biodiversity that can be measured in terms of:

- Impacts on aquatic community structure through changes in predator and prey population

- $\quad$ Changes in host abundance

- Reduction of intra specific genetic variation

- $\quad$ Possible extinction of species

There seems to be an urgent need to broaden the base of fisheries economics to meet the dynamic needs of fishing industry in respect of the following and the other related areas.

- $\quad$ Planning and management of fisheries.

- Economics (economic analysis) of fish culture, composite fish culture and integrated fish farming in respect of total investment, net production and profit (net income).

- $\quad$ Fish marketing and related problems.

- Socio-economic study of fishermen families.

- Manufacturing costs of crafts and gear and expenditure on fishing both in terms of human resources and other consumables.

- $\quad$ Economics of processing of fishery products and their export potential and related problems.

\section{Conclusion}

The socio-economic circumstances of small scale aquaculture holders are poor. Diseases are one of the constraints of fish farming in the country. Considering the above following recommendations should be made:

- $\quad$ Awareness of disease problems of small scale aquaculture needs to be raised.

- $\quad$ Reports of any kind of mass mortality should reach to nearby fishery officer as soon as possible.

- $\quad$ Provide a range of services and assistance such as generalized support services and confirmatory diagnosis of both current and newly emerging diseases.

- $\quad$ Facilitate research and act as contact centers for advice and training.

\section{References}

[1] M. Kottelat, and T. Whitten, Freshwater biodiversity in Asia with special reference to fish; in World Bank Techical Paper No. 343 (Washington, DC: The World Bank), 1996, 17-22.

[2] Y.S Yadav and R. Chandra, Some threatened carps and cat fishes of Brahmaputra river system; in Threatened fishes of India (eds) PV Dehadrai, P Das and SR Verma (Muzaffarnagar: Natcon Publication), 1994, pp 45-55

[3] U.C. Goswami, S.K. Basistha, D. Bora, K. Shyamkumar, B.Saikia and K.Changsan, Fish diversity of North East India, inclusive of the Himalayan and Indo Burma biodiversity hotspots zones: A checklist on their taxonomic status, economic importance, geographical distribution, present status and prevailing threats, International Journal of Biodiversity and Conservation, 4(15), 2012, 592-613.

[4] H.H. Williams and A. Jones, Parasitic worms of fish (London: Taylor and Francis, 1994), 593.

[5] M.K. Das, Outbreak of the fish disease, epizootic ulcerative syndrome in India - an overview: In: R.J. Roberts, B. Campbell and I.H. MacRae, (eds.) ODA Regional Seminar on Epizootic Ulcerative Syndrome, at the Aquatic Animal Health Research Institute, Bangkok, Thailand, 1994, 21-35.

[6] S.S.Khanna and H.R.Singh, A Text Book of Fish Biology and Fisheries(Narendra Publishing House:Delhi, 2003).

[7] V.V. SugunanandB.K.Bhattacharjya, Ecology and fisheries of beels in Assam, Central Inland Fisheries Research Institute (CIFRI) Bull.No.104, 2000, 64 
[8] Melba G. Bondad-Reantaso, Rohana P. Subasinghe, J. Richard Arthur, Kazuo Ogawac, SupraneeChinabut, Robert Adlarde, Zilong Tan, Mohamed Shariff, Disease and health management in Asian aquaculture, Elsevier :Veterinary Parasitology, doi:10.1016/j.vetpar,2005, VETPAR-3314; No of Pages 24.

[9] J.R. Arthur and R.P. Subasinghe, Potential adverse socio-economic and biological impacts of aquatic animal pathogens due to hatchery based enhancement of open-water system and possibilities for their minimization, 113-126. In: J.R. Arthur, M.J. Phillips, R.P. Subasinghe, M.B. Reantaso, I.H. MacRae, (Eds.) Primary Aquatic Animal Health Care in Rural Small-scale, Aquaculture Development (FAO Fish. Tech. Pap, 2002) 